Résumés des conférences et travaux

\title{
Philologie du bouddhisme chinois
}

\section{Liying Kuo}

\section{(2) OpenEdition \\ Journals}

Édition électronique

URL : https://journals.openedition.org/ashp/2006

DOI : $10.4000 /$ ashp.2006

ISSN : 1969-6310

Éditeur

Publications de l'École Pratique des Hautes Études

\section{Édition imprimée}

Date de publication : 1 septembre 2017

Pagination : 374-379

ISSN : 0766-0677

\section{Référence électronique}

Liying Kuo, "Philologie du bouddhisme chinois », Annuaire de l'École pratique des hautes études (EPHE), Section des sciences historiques et philologiques [En ligne], 148 | 2017, mis en ligne le 04 octobre 2017, consulté le 06 juillet 2021. URL : http://journals.openedition.org/ashp/2006 ; DOI : https://doi.org/ 10.4000/ashp.2006 


\title{
PHILOLOGIE DU BOUDDHISME CHINOIS
}

\author{
Directeur d'études à l'EFEO : M Me Liying Kuo
}

Programme de l'année 2015-2016 : Vision, méditation et purification dans le bouddhisme des $V^{\mathrm{e}}-V \mathrm{I}^{\mathrm{e}}$ siècles en Chine (suite et fin).

Le Dafangdeng tuoluoni jing 大方等陀羅尼經, “Grand vaipulya-dhāran̄isūtra » que nous avons étudié ces deux dernières années réserve bien des surprises. Le sūtra fut l'objet d'études par Paul Swanson (2000) et Koichi Shinohara (2012). Ces deux chercheurs ont essayé de retrouver le titre d'origine du sūtra en sanskrit et les noms sanskrits de certains personnages qui jouent un rôle central dans des épisodes narratifs du sütra. Mais leurs restitutions de l'original sanskrit ne sont pas les mêmes. Il nous semble qu'ils ne se sont jamais demandé si le sūtra était authentique ou non. Or certains passages du texte s'expliquent uniquement à partir du chinois. Par exemple le ou les rédacteurs du sütra consacrent un très long discours à expliquer le nom d'un ermite condamné dans l'enfer Avīci, Poyu 婆蒱 (T. 1339, 21 : 643644). Le sūtra donne dix interprétations de chacun des deux mots, po et $y u$, qui ne se comprennent qu'en contexte chinois et n'ont rien à voir avec le nom sanskrit. Car le personnage est connu sous son nom sanskrit, Vasu, transcrit généralement Posou 婆 數 (Paul Demiéville, « Baso / Vasu », Hôbôgirin I : 57b-58a; T. 1509, 25 : 76a-b et Étienne Lamotte, Traité de la grande vertu de sagesse, I : 165-168). Poyu 婆荀 fut probablement une erreur de scribe pour Posou 婆數. Mais les interprétations linguistiques du sūtra n'ont pas plus de rapport avec Posou qu'avec Poyu ou Vasu.

En étudiant les copies manuscrites de Dunhuang datées du début du $\mathrm{VI}^{\mathrm{e}}$ siècle, nous constatons l'existence de variantes non négligeables par rapport aux versions imprimées et éditées à partir du XII ${ }^{\mathrm{e}}$ siècle (Canon édition coréenne, éditions des dynasties des Song, Yuan et Ming) utilisées pour le Canon du Taishō. De plus, on a découvert dans un ancien monastère situé dans le sud de Shanxi une stèle érigée en 560 par une association bouddhiste locale réunissant des religieux et des fidèles laïcs, qui donne les noms de onze des douze scènes oniriques, dites rois des rêves (shier mengwang 十二夢王), énumérées dans le sūtra, mais avec des variantes par rapport aux manuscrits de Dunhuang et aux textes imprimés du XII ${ }^{\mathrm{e}}$ siècle. L'ensemble de ces variantes nous amène à nous demander à nouveau si ce dhâraṇi-sūtra, censé traduit du sanskrit au début du v v siècle à Zhangye 張掖 (dans l'actuelle province du Gansu), capitale du royaume des Liang du Nord, nouvellement établi par un clan Hu, Juqu 沮 渠, n’est pas en réalité un apocryphe. Les manuscrits de Dunhuang montrent que le texte du sūtra n'était pas tout à fait fixé même au début du $\mathrm{VI}^{\mathrm{e}}$ siècle, soit un siècle après la date donnée pour sa traduction.

Nous avons étudié spécialement une courte portion de texte qui se trouve uniquement à la fin de deux copies de Dunhuang, avant le titre final et le colophon. Il s'agit des manuscrits S. 6727 et S. 1524 datés respectivement de 514 et 521. Le colophon du S. 6727 indique que la copie est l'œuvre d'un copiste officiel du bureau de copie de sütra-s du canton de Dunhuang. Cette portion de texte est totalement inédite. En voici la traduction : 
Aujourd'hui, le $7^{\mathrm{e}}$ jour du onzième mois lunaire, Moi, un biqiu 比丘 (mot transcrit du sanskrit bhiksu, «moine ») X, je suis le maître-enseignant (jiaoshou shi 教授師) du disciple laïc homme (youposai 優婆塞, upāsaka) ou femme (youpoyi 優婆夷, up $\bar{a}$ sika $) \mathrm{Y}$, qui m'invite à être son maître-enseignant. J'accepte d'être pour toujours son maitre-enseignant. Les Buddhas des dix directions doivent témoigner, les honorables Prajñā-sūtra-s (zunjing Poruo 尊經波若) doivent témoigner, les moines qui ont déjà atteint la vérité (yingzhen seng 應真僧) doivent témoigner que le disciple Y m'accepte pour maître-enseignant. Du $8^{\mathrm{e}}$ jour au $15^{\mathrm{e}}$ jour [de ce mois] il (ou elle) pratique sous ma conduite la cérémonie zizi 自恣 (《auto-indulgence»). [La même formule] doit être prononcée une deuxième et une troisième fois : les Buddhas des dix directions doivent témoigner, les honorables sūtra-s (zunjing 尊經) doivent témoigner, les moines qui ont atteint la vérité doivent témoigner que le disciple $\mathrm{Y}$ m'accepte pour maître-enseignant. Toi, le pratiquant, écoute attentivement de tout cœur, mémorise, pratique, lis et récite [ce Grand vaipulya-dhāraṇi-sūtra]. Du $8^{\mathrm{e}}$ jour au $15^{\mathrm{e}}$ jour, tu dois écouter attentivement. [Je vais alors] expliquer le sens de l'acte rituel (jiemo fa 羯磨法, karma-dharma).

À ce moment-là, Mañjuśrī se lève de son siège, l'épaule droite découverte, et s'adresse au Buddha: Vénéré du Monde, durant l'époque où le Buddha est présent dans ce monde, ou a quitté ce monde, les hommes et femmes de bien qui souhaitent observer, réciter et pratiquer le Grand vaipulya-dhāraṇi-sūtra, doivent psalmodier les [vingtquatre] stances heptasyllabiques qui suivent :

Mañjuśrī prêche le bon dharma pour qu'il se diffuse dans le continent du Jambudvīpa. Dans cette aire de bodhi [toi, le pratiquant] tu dois pratiquer assidûment en gardant ta pensée en un seul lieu sans te déconcentrer.

Tu verras ainsi le bodhisattva [Mañjuśrī] et tous les Buddhas.

[Mañjuśrī] prêche [le dharma] à celui qui comprend déjà [la nature des choses] et peut retenir ainsi dans son cœur ce bon dharma enseigné par tous les Buddhas des Trois Époques.

Dans l'aire de bodhi, lors de sa contemplation le pratiquant aperçoit [le bodhisattva] Maitreya et entend son enseignement.

Il [Maitreya] convertit les êtres pour qu'ils quittent [leur existence dans] les trois mondes [de forme, de désir et de non-forme]

et consolident leurs forces pour échapper à la chaîne de causation dans ces trois mondes.

Ce dhārañi-sütra est extraordinaire,

c'est pourquoi il est pratiqué en permanence dans le Jambudvīpa.

Il (Mañjuśrín) voit que les êtres doivent être convertis.

Il va chez eux prêcher le bon dharma.

Il y a d'immenses souffrances dans l'enfer Avicī.

Elles vous affligent sans vous laisser un seul moment de répit.

Même si vous tentiez de les fuir, il n'y a nulle part où vous échapper.

Le monde des Buddhas est magnifique.

Vous, les gens, pour quelles raisons endureriez-vous de telles souffrances?

Le dhārañi-sütra peut vous délivrer de ces souffrances.

Il guide tous les êtres jusqu'à l'autre rive (bi'an 彼岸).

L'ensemble d'activités évoquées dans ce texte commence au $7^{\mathrm{e}}$ jour du onzième mois lunaire, le jour où le moine accepte d'être le maître-enseignant d'un fidèle laïc, homme ou femme. Il prononce trois fois la même formule en invitant les Buddhas, les 
Prajñā-sūtra-s et les moines qui ont déjà atteint la vérité (arhant-s ou bodhisattva-s) à être les témoins du rite de transmission. Les honorables Prajñā-sūtra-s se retrouvent dans le quatrième juan du sütra qui décrit comment « garder les préceptes » (hujie 護 戒). Il y est dit qu'un ou une novice, un ou une fidèle qui transgresse « les interdictions / préceptes » (zhujin 諸禁), doit inviter un bhikșu connaissant tous les vinaya-s et, en face de la statue (xiang xingxiang qian 向形像前) [du Buddha], ou devant les honorables Prajñā-sūtra-s (zunjing Panruo qian 尊經波若前) avouer ses fautes à ce bhikșu. Ce dernier doit lui enseigner la méthode de se purifier selon les codes des vinaya-s (jinlü zhifa 淨律之法; manuscrit de Dunhuang dans la collection de l'université normale du Nord-Ouest cote 017 : col. 41-44; T. 1339, 21 : 657a29-b3). Ailleurs dans le sūtra, il est dit qu'à défaut d'avoir une image du Buddha, on peut utiliser un exemplaire du Prajñā-sūtra personnalisé et divinisé. Mais dans cette courte portion de texte de Dunhuang les Prajñāa-sūtra-s prennent place à côté des Buddhas et des moines. Le précepteur invite ainsi les trois joyaux du bouddhisme, Buddha, Dharma (Prajñā-sütra) et Samigha (communauté de moines) à être présents à la cérémonie. La transmission des préceptes du maître-moine au disciple laïc est ainsi aussi valide que le rite de prendre les trois refuges dans le bouddhisme et la cérémonie de l'ordination des moines.

C'est à partir du deuxième jour et durant huit jours, c'est-à-dire du $8^{\mathrm{e}}$ jour au $15^{\mathrm{e}}$ jour du onzième mois, que le moine précepteur invite son disciple laïc à pratiquer la cérémonie $z i z i$ 自恣. Le terme $z i z i$ est l'un des termes chinois utilisés pour traduire le sanskrit pravāraṇā, qui implique un rite de purification célébré à la fin de la retraite des religieux en Inde. Les dates des $8^{\mathrm{e}}$ et $15^{\mathrm{e}}$ jours sont aussi indiquées dans le premier juan du sütra. Il y est dit que durant ces sept jours (sic!) un pratiquant qui souhaite observer le vrai dharma des Buddhas doit pratiquer le jeûne. Trois fois par jour il doit se baigner et revêtir un vêtement propre. Il doit aussi faire fabriquer une statue du Buddha et un parasol aux cinq couleurs. Il récite ensuite vingt fois la dhāraṇi du sūtra en faisant chaque fois la circumambulation autour de la statue du Buddha. Ainsi les fautes même les plus graves, mêmes les cinq péchés irrémissibles (wuni 五逆), seront éliminés. Mais le sūtra insiste aussi sur la nécessité de d'abord confesser les éventuelles transgressions des préceptes prescrits pour les différents statuts religieux (moine, nonne, novice, laïc) et notamment des vingt-quatre préceptes de bodhisattva, qu'il énumère (S. 1524, S. 6727, S. 4248; T. 1339, 21 : 645b-646b7).

Dans ce court texte inédit intercalé dans S. 1524 et S. 6727 la nature des préceptes n'est pas signalée, mais il est clair que ce sont les vingt-quatre préceptes de bodhisattva énumérés dans le premier juan du sūtra indiqué ci-dessus. La deuxième partie de ce texte inédit est rédigée en stances heptasyllabiques à psalmodier; les deux bodhisattva-s, Mañjuśrī et Maitreya, y sont les maîtres-enseignants mystiques. Avec le Buddha Sākyamuni, ils forment donc les «trois maîtres » (sanshi 三師) du rite de transmission des préceptes de bodhisattva dont il est question pour la première fois dans l'un des plus fameux sütra-s traitant de la contemplation, le Guan Puxian pusa xinfa jing 觀普賢菩薩行法經, «Sūtra de la contemplation des actes du bodhisattva Samantabhadra » (T. 277, $9: 393$ c) qui aurait été traduit d'un texte indien par un moine cachemirien, Tanwumiduo 昙無密多 (Dharmamitra, 356-442). La réception des préceptes devant ces trois maîtres et des témoins est attestée sur des certificats de 
réception des préceptes de bodhisattva trouvés notamment à Dunhuang, par exemple les manuscrits S. 2851 et S. 1780. Sur les certificats, de plus, les grands bodhisattva-s sont désignés comme les compagnons d'études. Il arrive que parfois on évoque le Buddha Amitābha à la place de Mañjuśrī (P. 3207, S. 4482, S. 6264).

Comme indiqué plus haut ce texte ne se trouve que sur les deux copies du premier juan du Grand vaipulya-dhāraṇi-sütra, qui sont des copies officielles du canton de Dunhuang. On doit donc se demander si cette portion du texte n'a pas été utilisée à Dunhuang au $\mathrm{VI}^{\mathrm{e}}$ siècle à la fin de la retraite de la communauté religieuse, au onzième mois lunaire, quand les moines organisaient une cérémonie pour les laïcs. Ils y acceptaient d'être leurs précepteurs et de les guider dans la pratique du rite de purification. Il est probable qu'alors ils enseignaient ce dhārañi-sūtra, prescrivaient les vingt-quatre préceptes de bodhisattva du sütra, et faisaient psalmodier les vingtquatre stances heptasyllabiques copiées dans la partie finale de S. 1524 et S. 6727. Très récemment, $\mathrm{M}$. Wang Huimin, de la Dunhuang Academy, a proposé de voir dans une peinture du mur sud de la grotte 320 de Mogao qui montre le Buddha prêchant et, dessous, les bodhisattva-s assis chacun sur une fleur de lotus flottant, une illustration d'une des scènes du Grand vaipulya-dhārañi-sūtra. Mais la peinture en question est en mauvais état et ne semble pas comporter de détails spécifiques se rapportant au Grand vaipulya-dhāraṇi-sūtra.

La deuxième partie du cours a été consacrée à la lecture de manuscrits de Dunhuang datés des $\mathrm{V}^{\mathrm{e}}-\mathrm{VI}^{\mathrm{e}}$ siècles en rapport avec les pratiques de réception des préceptes. Le manuscrit S. 797, considéré comme le plus ancien manuscrit daté de Dunhuang car il porte la date 406, serait selon l'étude de Yabuki Keiki et Tsukamoto Zenryū, une copie des $27^{\mathrm{e}}$ et $28^{\mathrm{e}}$ rouleaux de la traduction chinoise du Sarvāstivāda-vinaya. Ce texte a été traduit dès 404, d'abord par Furuoduoluo 弗若多羅 (Punyatara? ca 399415) et Dharmaruci (actif en 405), puis remanié et complété par Kumārajīva (mort en 409 ou 413). La date de 406 ferait de la copie de Dunhuang la version primitive de la traduction, antérieure à l'intervention du grand maître Kumārajīva (T. 1435). En fait, il existe des différences entre la copie de Dunhuang et le texte édité dans le Canon du Taishō. Deux textes se trouvent à la fin de S. 797. Le premier est un court texte intitulé Shousuiwen 受歲文, 《Texte pour recevoir la [nouvelle] année » (shousui est une autre expression chinoise pour traduire le sanskrit pravāranāa), c'est donc un formulaire de la cérémonie pravāraṇa qui se célèbre une fois par an à la fin de la retraite saisonnière des moines. Le biqiu (bhikșu) invite les membres de l'assemblée à énumérer toutes leurs fautes, vues, soupçonnées et entendues (jianzui 見罪, yizui 疑罪, wenzui 聞罪) et à les confesser. Comme d'habitude, il est indiqué que le formulaire doit être prononcé trois fois. Le deuxième texte indique une date du $5^{\mathrm{e}}$ jour du douzième mois de la première année Jianchu, c'est-à-dire le 16 janvier 406, le jour où un bhikṣu nommé Deyou 德祐 a reçu les préceptes complets (nécessaires pour un moine en titre) au sud de la ville de Dunhuang devant le maître (heshang 和上, traduisantt le sanskrit upadhyaya) Faxing 法性, le maître de préceptes (jieshi 戒師) Baohui 寶慧 et le maître-enseignant (jiaoshi 教師) Huiying 惠穎. À ce moment-là étaient présents dans la salle d'ordination Daofu 道輔, Huiyu 惠御, etc., en tout douze personnes venues à Dunhuang observer « la retraite de l'été » (xia anju 夏安 居). Il s'agit donc d'une ordination qui eut lieu à la fin de la retraite de la communauté 
bouddhiste à Dunhuang en hiver, bien que le moine Deyou parle de « la retraite de l'été », expression indienne. La période de retraite des religieux change en fonction du climat du pays où ils se trouvent. Les vinaya-s, qui sont tous traduits de textes indiens, utilisent l'expression xia anju 夏安居 pour traduire l'indien varșa « la retraite de la saison des pluies », l'été en Inde, mais pas en Chine. Cependant quelques rédactions chinoises parlent aussi de 《la retraite de l'hiver»(dong anju 冬安居), mais c'est beaucoup plus rare. Il y a peut-être eu parfois deux retraites par an, une en été et une en hiver. Il se peut que l'expression xia anju (varșa de l'été) ait été employée pour indiquer la retraite des religieux bouddhistes en général, sans implication sur la saison réelle, comme le montre le texte du moine Deyou à Dunhuang dont l'ordination complète se fit devant les trois maîtres en chair et en os. Les douze autres moines ont probablement servi de témoins. Il n'y a que dans les traités des Yogācārya-bhūmi et Bodhisattva-bhūmi qu'un aspirant à la condition de bodhisattva est autorisé à recevoir les préceptes de bodhisattva par lui-même, seul, devant la statue du Buddha, quand il n'existe dans un périmètre de mille lieues aucun maître qualifié pour lui conférer les préceptes de bodhisattva. Les maîtres mystiques, Buddhas et bodhisattva-s, prennent ainsi le rôle de trois maîtres humains et garantissent que l'aspirant en question a reçu les préceptes de bodhisattva et peut se déclarer « disciple [qui a reçu] les préceptes de bodhisattva»(Pusajie dizi 菩薩戒弟子). Il pratique désormais 《les actes de bodhisattva».

Un autre manuscrit de Dunhuang, le P. 2196, quant à lui, expose le caractère grandiose des préceptes de bodhisattva et la méthode pour les recevoir. Le texte du manuscrit est complet, mais le titre manque, au début tout au moins car à la fin du texte on lit: Chujia ren shou pusa jiefa juan diyi 出家人受菩薩戒法卷第一, « Méthode pour les religieux qui veulent recevoir les préceptes de bodhisattva, juan premier ». Un colophon indique que [le texte] fut écrit au cinquième mois, l'été de l'an jihai, de la $18^{\mathrm{e}}$ année Tianjian (519) des Grands Liang sur ordre de l'empereur Wu des Liang (r. 502-549), un fervent bouddhiste. Le manuscrit a été écrit par un laïc nommé Dai Mengtong 戴萌桐 et relu par un autre laïc, Gao Xianzhi 睪岀之. Le texte a été mis en pratique par Huiming 慧明, moine du monastère Waguan 瓦官. Selon la biographie du moine Huiyue 慧約 (452-535), dans la 11e année Tianjian (512), l'empereur aurait sélectionné dans toute la littérature bouddhique et composé lui-même « des chapitres de préceptes » (jiepin 戒品). Les préceptes sont distinctement énumérés l'un après l'autre, puis vient la description détaillée du rituel. L'empereur fit également dresser un autel de forme circulaire pour ce rite. Au $8^{\mathrm{e}}$ jour du quatrième mois de la $18^{\mathrm{e}}$ année Tianjian (519) l'empereur lui-même reçut les préceptes de bodhisattva (T. 2060, 50 : 469b). Il est donc possible que l'empereur Wu soit l'auteur de la « Méthode pour les religieux qui veulent recevoir les préceptes de bodhisattva » retrouvée à Dunhuang dont la seule copie existante fut faite sur son ordre en 519, environ un mois après sa propre ordination de bodhisattva. Puisqu'après le titre de fin il est indiqué " premier juan », le texte devait en comporter au moins deux. M. Ochiai Toshinori a examiné une copie manuscrite japonaise de l'époque Heian (794-1185) intitulée le Zaikenin fusetsuho, kan dai-shichi 在家人布薩法卷第七, “ Méthode pour les laïcs pour observer la pratique du poșadha, septième juan ». Le poșadha, transcrit en chinois par busa 布薩, fusetsu en prononciation japonaise, est la réunion des moines 
tous les quinze jours afin de se purifier en confessant les fautes commises. La pratique $\mathrm{du}$ busa fut adoptée très tôt, dès le $\mathrm{VI}^{\mathrm{e}}$ siècle, par les lettrés bouddhistes chinois. Ils la désignaient par zhai 齋, « jeûne». Mais ici encore le manuscrit japonais ne comporte que le septième juan. Selon l'analyse de M. Ochiai, ce septième juan contient beaucoup de similitudes avec le premier juan de Dunhuang. Peut-être faisait-il partie d'un manuel de réception de préceptes de bodhisattva en plusieurs juan. 